\section{Gammopathie, monoklonale}

H. Baum

Institut für Laboratoriumsmedizin, Mikrobiologie und

Blutdepot, Regionale Kliniken Holding RKH GmbH,

Ludwigsburg, Deutschland

Englischer Begriff monoclonal gammopathy

Definition Zunahme eines antigenetisch, strukturell und funktionell einheitlichen Immunglobulins oder Immunglobulinbruchstückes, die exzessive Ausmaße erreichen kann.

Beschreibung Die monoklonale Gammopathie ist eine exzessive Vermehrung eines antigenetisch, strukturell und funktionell einheitlichen Immunglobulins oder eines Immunglobulinbruchstückes bei Leukosen der B-Zellreihe. Die Benennung bezieht sich auf die auffällige, meist spitzgipflige Erhöhung der Gamma-Fraktion bzw. zwischen der Beta- und Gamma-Fraktion in der Eiweißelektrophorese bei diesen Erkrankungen. Aufgrund des exprimierten Immunglobulins werden die monoklonalen Gammopathien den Klassen IgG, IgA, IgM, IgD und IgE sowie den Leichtkettentypen Kappa und Lambda zugeordnet. Die monoklonalen Gammopathien werden in symptomatische und maligne Formen, fakultative Formen, die monoklonale Gammopathie unbestimmter Sig- nifikanz (MGUS) und passagere Formen eingeteilt (s. Tabelle).

Einteilung der monoklonalen Gammopathien (nach Thomas 1995):

\begin{tabular}{|c|c|c|}
\hline \multicolumn{3}{|l|}{ Gammopathieformen } \\
\hline $\begin{array}{l}\text { Monoklonale } \\
\text { symptomatische }\end{array}$ & $\begin{array}{l}\text { Fakultativ } \\
\text { monoklonale }\end{array}$ & $\begin{array}{l}\text { Monoklonale } \\
\text { unbekannter } \\
\text { Signifikanz } \\
\text { (MGUS) }\end{array}$ \\
\hline $\begin{array}{l}\text { Obligat einhergehende } \\
\text { hämatologische } \\
\text { Systemerkrankungen: } \\
\text { - Multiples Myelom } \\
\text { - Morbus Waldenström } \\
\text { (IgM-Paraproteinämie) } \\
\text { - } \\
\text { Kälteagglutininkrankheit } \\
\text { - Schwerkettenkrankheit }\end{array}$ & $\begin{array}{l}\text { Begleitend bei } \\
\text { anderen Non- } \\
\text { Hodgkin- } \\
\text { Lymphomen } \\
\text { (z. B. CLL) }\end{array}$ & $\begin{array}{l}\text { Keiner } \\
\text { Grunderkrankung } \\
\text { zuzuordnen und } \\
\text { ohne klinische } \\
\text { Symptomatik, tritt } \\
\text { mit zunehmender } \\
\text { Häufigkeit mit } \\
\text { steigendem } \\
\text { Lebensalter auf } \\
\text { (3\% bei über } \\
80 \text {-Jährigen, >6\% } \\
\text { bei über } \\
\text { 90-Jährigen) }\end{array}$ \\
\hline
\end{tabular}

\section{Literatur}

Thomas L (1995) Immunsystem - Monoklonale Gammopathien. In: Greiling H, Gressner AM (Hrsg) Lehrbuch der Klinischen Chemie und Pathobiochemie, 3. Aufl. Schattauer Verlag, Stuttgart/New York, S $1178-1179$ 